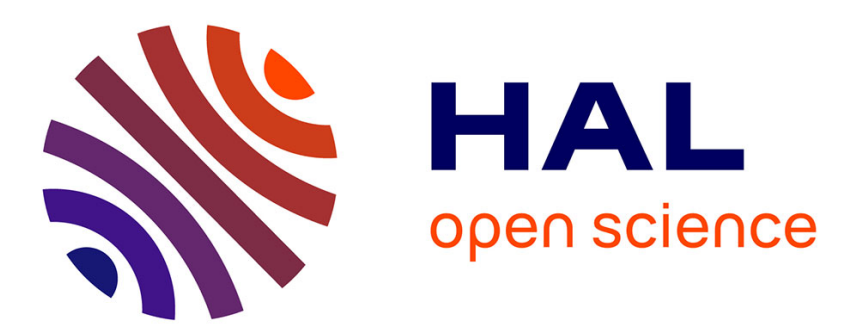

\title{
Découverte d'un diatrème de kimberlite diamantifère à Séguéla en Côte-d'Ivoire.
}

\author{
André Pouclet, Marc Allialy, Bertin Daouda-Yao, Botty Esso
}

\section{To cite this version:}

André Pouclet, Marc Allialy, Bertin Daouda-Yao, Botty Esso. Découverte d'un diatrème de kimberlite diamantifère à Séguéla en Côte-d'Ivoire.. Comptes rendus de l'Académie des sciences. Série IIa, Sciences de la terre et des planètes, 2004, 336, pp.1, 9-17. 10.1016/j.crte.2003.10.012 . hal-00023894

\section{HAL Id: hal-00023894 \\ https://hal-insu.archives-ouvertes.fr/hal-00023894}

Submitted on 19 May 2006

HAL is a multi-disciplinary open access archive for the deposit and dissemination of scientific research documents, whether they are published or not. The documents may come from teaching and research institutions in France or abroad, or from public or private research centers.
L'archive ouverte pluridisciplinaire HAL, est destinée au dépôt et à la diffusion de documents scientifiques de niveau recherche, publiés ou non, émanant des établissements d'enseignement et de recherche français ou étrangers, des laboratoires publics ou privés. 


\title{
Découverte d'un diatrème de kimberlite diamantifère à Séguéla en Côte-d'I voire
}

\section{Discovery of a diamond-bearing kimberlite diatreme at Séguéla in I vory Coast}

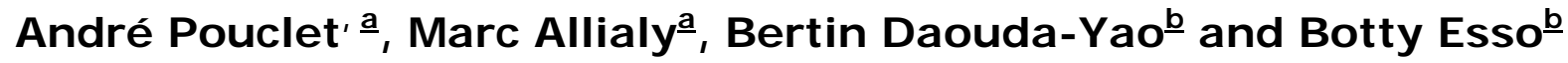 \\ a Institut des sciences de la Terre d'Orléans, UMR 6113, université \\ d'Orléans, 45067, Orléans cedex 2, France \\ ${ }^{b}$ Direction de la Géologie, ministère des Mines et de l'Énergie, Abidjan, \\ Côte d'Ivoire
}

\section{Résumé}

La région de Séguéla, au centre-ouest de la Côte-d'Ivoire, est connue pour son champ diamantifère alimenté par des dykes de kimberlite et de lamproïte. Ces dykes, non déformés et non métamorphisés, recoupent les formations du Birimien. Leur orientation $N 170^{\circ}$ est contrôlée par le contact structuré entre les lithosphères archéenne et paléoprotérozoïque du craton ouest-africain. La découverte d'un diatrème avec les brèches de cheminée et les sédiments du maar préservés sous les colluvions actuelles atteste l'âge géologique relativement récent (Crétacé ?) de l'activité kimberlitique

\section{Abstract}

The Séguéla area in Ivory Coast is known for its diamond-bearing field related to dykes of kimberlite and lamproite. These dykes, devoid of any deformation and metamorphism, crosscut the Birimian formations. Their $\mathrm{N}$ $170^{\circ}$ orientation is controlled by the tectonized contact between the Archean and the Paleoproterozoic shields of the West-African craton. Discovery of a diatreme, with its pipe breccias and well-preserved maar sediments, below the present-day colluvia, attests for the probable recent geological age (Cretaceous?) of the kimberlitic activity.

Mots-clé: kimberlite; diamant; diatrème; Côte-d'IvoireMots-clé: kimberlite; diamond; diatreme; Ivory Coast

\section{Geological setting and kimberlitic features}

An important diamond-bearing field is located in the central-western part of Ivory Coast, $30 \mathrm{~km}$ north of Séguéla [ $\underline{1}$ and $\underline{9}$ ] ( Fig. 1). Diamonds, 
averaging $0.3 \mathrm{ct}$, are disseminated into eluvia, colluvia and alluvia and originated from the two main kimberlitic dykes of Bobi and Toubabouko. The richer parts of the field were formerly mined by two companies. Now, only individual diggers are working in all the area. The dykes, trending $\mathrm{N}$ $170^{\circ}$, crosscut the granitic plutons and amphibolites of the Palaeoproterozoic Birimian formations of the West-African craton. One granite is dated at $2.091 \pm 8 \mathrm{Ma}$ [10]. The dyke of Bobi (BD) is $2.5 \mathrm{~km}$ long and 25 to $50 \mathrm{~cm}$ wide. Length of the dyke of Toubabouko (TD) reaches $4.5 \mathrm{~km}$ and its wideness, $80 \mathrm{~cm}$ to $1 \mathrm{~m}$. In the northern part of this dyke, a particularly enriched zone was recently dug in a large area in a $80-\mathrm{m}$-indiameter and $30-\mathrm{m}$-deep pit ( $8^{\circ} 15^{\prime} 22$, W 6 $\left.6^{\circ} 37^{\prime} 57\right)$. Here, we discovered (9 February 2002) a typical kimberlitic diatreme with its complete lithostructural features (Fig. 2).

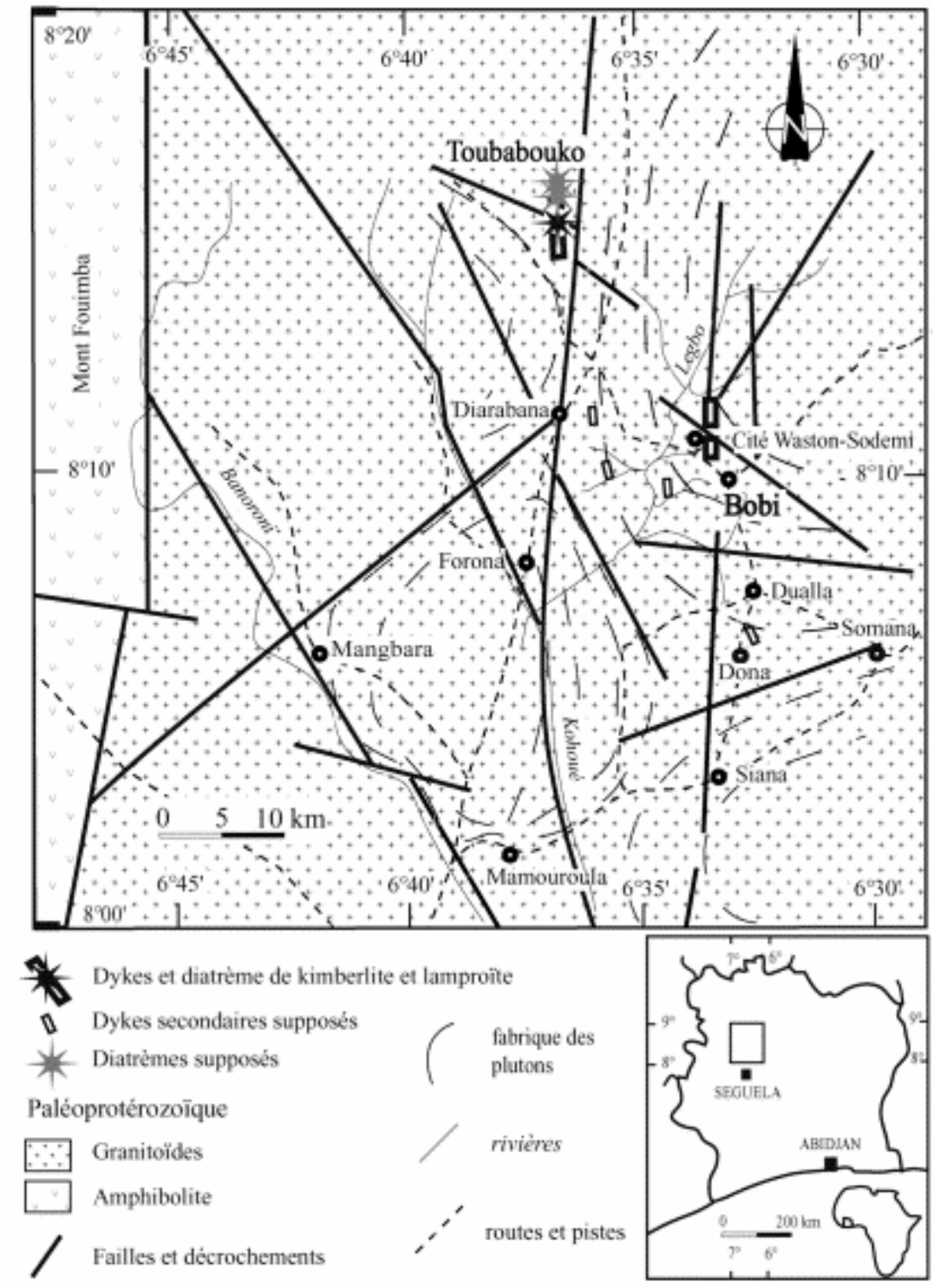

Fig. 1. Carte de localisation des dykes kimberlitiques de Bobi et de Toubabouko au nord de Séguéla (Côte-d'Ivoire).Location map of the kimberlitic dykes of Bobi and Toubabouko, north of Séguéla (Ivory Coast). 


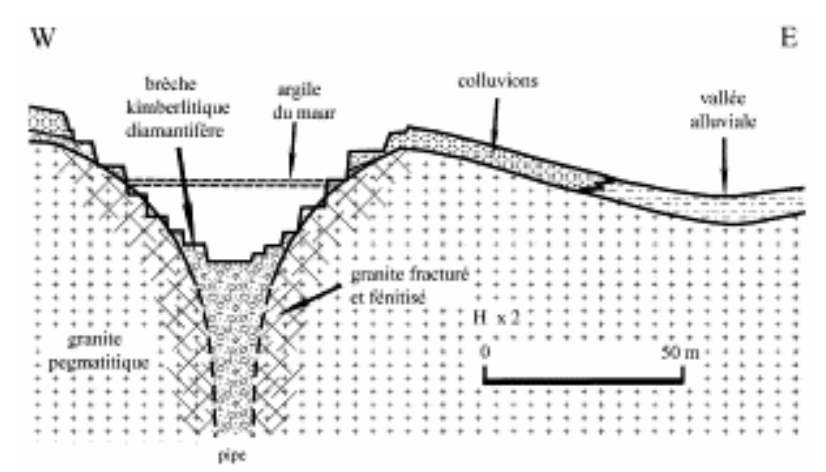

Fig. 2. Coupe schématique du diatrème de Toubabouko.Schematic crosssection of the Toubabouko diatreme.

The walls of the pit exhibit a pile of breccias consisting of decimetric and more or less rounded blocks of aphyric kimberlite highly altered into talc and celadonite. Blocks are cemented by accumulation of 2-mm to $2-\mathrm{cm}$ fragments of pegmatitic granite material with mono- and polycrystalline debris of quartz, potassic and sodic feldspar, muscovite and rare biotite. This cement reaches 30 to $40 \%$ of the breccias. In the deeper part, there are larger blocks, around $1 \mathrm{~m}$, embedded in stratified granite clasts. No xenoliths were found. At the upper middle bench of the eastern wall, a finely stratified and horizontal sedimentary clay layer overlies the lower breccias and is slightly curved against the breccias of the flank. It is made of kaolinite millimetric beds including silty-sized quartz, potassic and sodic feldspar angular grains, iron hydroxides and alternation of cumulated flakes of phlogopite altered into smectites (for the chemical composition of the clay layer, see Table 1). This layer corresponds to a lacustrine sediment. If we extend to the centre axis of the pit of the strata preserved on the bench, we reconstitute a 1.5- to 2.5-m-thick clay layer, which was in the middle part of the breccia area, and, before the digging, below the overlying colluvia. This kind of sediment was deposited in a pond of a kimberlitic breccia crater, i.e., in a maar. At some hundred of metres in the northern prolongation of the dyke, two additional occurrences of kimberlitic breccia are partly exposed. That means the dyke emplaced at a very shallow level and evolved to explosive diatremes at its northern end. 


\begin{tabular}{|c|c|c|c|c|c|}
\hline & $\begin{array}{c}\text { Argile } \\
\text { Maar (MA-7) }\end{array}$ & $\begin{array}{c}\text { Kimberlite s.5. } \\
\text { Toubabouko (K-12) }\end{array}$ & $\begin{array}{l}\text { Kimberlite noduleuse } \\
\text { Toubabouko (K-3b2) }\end{array}$ & $\begin{array}{l}\text { Lamproite } \\
\text { Bobi (K-11) }\end{array}$ & $\begin{array}{c}\text { Lamproite } \\
\text { Taylor et al. [15] }\end{array}$ \\
\hline $\mathrm{SiO}_{2}(\%)$ & 47,01 & 49,75 & 55,60 & 36,95 & 41,8 \\
\hline $\mathrm{TiO}_{2}$ & 1,12 & 2,61 & 1,52 & 3,98 & 5,9 \\
\hline $\mathrm{Al}_{2} \mathrm{O}_{3}$ & 24,91 & 4,35 & 1,61 & 7,11 & 3,5 \\
\hline $\mathrm{Fe}_{2} \mathrm{O}_{3}$ & 9,39 & 6,84 & 6,12 & 10,27 & 12,0 \\
\hline $\mathrm{MnO}$ & 0,05 & 0,11 & 0,05 & 0,14 & 0,1 \\
\hline $\mathrm{MgO}$ & 1,46 & 21,14 & 26,43 & 20,27 & 16,2 \\
\hline $\mathrm{CaO}$ & 0,57 & 5,48 & 1,31 & 8,10 & 8,3 \\
\hline $\mathrm{Na}_{2} \mathrm{O}$ & 0,52 & 0,24 & 0,00 & 0,72 & 1,3 \\
\hline $\mathrm{K}_{2} \mathrm{O}$ & 1,34 & 0,20 & 0,73 & 1,11 & 3,0 \\
\hline $\mathrm{P}_{2} \mathrm{O}_{5}$ & 0,12 & 1,16 & 0,64 & 1,36 & 3,6 \\
\hline PF (LOI) & 13,37 & 8,05 & 6,01 & 9,45 & - \\
\hline Total & 99,86 & 99,93 & 100,02 & 99,46 & 95,70 \\
\hline $\mathrm{Rb}$ (ppm) & 90,2 & 4,48 & 36,4 & 36,2 & \\
\hline $\mathrm{Ba}$ & 503 & 117 & 462 & 934 & \\
\hline $\mathrm{Th}$ & 15,2 & 16,1 & 10,2 & 24,7 & \\
\hline $\mathrm{Nb}$ & 23,8 & 199 & 86,9 & 282 & \\
\hline $\mathrm{Ta}$ & 1,51 & 11,6 & 5,13 & 16,3 & \\
\hline La & 92,3 & 259 & 79,8 & 340 & \\
\hline $\mathrm{Ce}$ & 158 & 481 & 183 & 597 & \\
\hline $\mathrm{Pr}$ & 19,1 & 55,4 & 23,7 & 69,5 & \\
\hline $\mathrm{Sr}$ & 151 & 883 & 407 & 1218 & \\
\hline $\mathrm{Nd}$ & 67,3 & 186 & 85,0 & 236 & \\
\hline $\mathrm{Zr}$ & 209 & 719 & 356 & 933 & \\
\hline Hf & 5,24 & 15,6 & 9,47 & 20,7 & \\
\hline $\mathrm{Sm}$ & 10,7 & 19,4 & 10,4 & 25,8 & \\
\hline $\mathrm{Eu}$ & 2,19 & 4,32 & 2,48 & 6,24 & \\
\hline $\mathrm{Gd}$ & 8,17 & 8,98 & 4,50 & 12,9 & \\
\hline $\mathrm{Tb}$ & 1,12 & 1,17 & 0,73 & 1,67 & \\
\hline Dy & 5,94 & 4,72 & 2,86 & 6,47 & \\
\hline $\mathrm{Y}$ & 32,8 & 12,9 & 9,71 & 20,3 & \\
\hline $\mathrm{Yb}$ & 2,54 & 0,80 & 0,67 & 1,10 & \\
\hline
\end{tabular}

Tableau 1. Analyses chimiques de l'argile du maar et des trois types pétrographiques de kimberlites. Analyses par ICP (majeurs et quelques mineurs) et ICP-MS (mineurs) au CRPG de Nancy. Analyse ancienne de lamproïte de Séguéla donnée par Taylor et al. [15]Chemical analyses of the clay of the maar and of the three kimberlite petrographical types. Analyses by ICP (major and some minor elements) and ICP-MS (minor elements) from CRPG of Nancy. Former analysis of Séguéla lamproite given by Taylor et al. [15]

\section{Composition of the kimberlitic rocks}

We distinguish three petrographical types: sensu stricto kimberlite, kimberlite enriched in olivine nodules, and micaceous kimberlite or lamproite. The common kimberlite is a fine-grained aphyric rock at the dyke margins, or moderately phyric inside the two main dykes and into the diatreme. Phenocrysts consist of olivine totally replaced by serpentine at depth (core samples) and by talc close to the surface. Matrix is made of tiny flakes of Ti-phlogopite $\left(\mathrm{TiO}_{2}=4.8-5.5 \%\right)$, magnetite grains, xenomorphic $\mathrm{Mn}$-ilmenite $(\mathrm{MnO}=10.9-18.7 \%)$, and accessory magmatic minerals: apatite, titanite, zircon, baddeleyite, monazite, priderite $(\mathrm{BaO}=2.2-3.8 \%)$, jeppeite $(\mathrm{BaO}=17.3-19.9 \%)$ and... diamonds. Abundant secondary mineralogy in the groundmass consists of $\mathrm{Mg}$-chlorite (penninite), saponite, celadonite, talc, anatase, iron hydroxides, calcite and quartz. In the middle part of the TD, the rock is highly enriched in 1 
to 10-mm-large nodules of olivine, also replaced by serpentine or talc, and contains some xenocrysts of $\mathrm{Mg}$-chromhercynite mantled by chromite and $\mathrm{Cr}$-magnetite. The micaceous kimberlite is characterized by the increasing size of the phlogopite flakes, the decreasing amount of olivine, and the occurrence of Ba-rich titanates. It is defined as lamproite when showing a typical lamprophyric texture. This facies is abundant in the $\mathrm{BD}$, and is also present in the TD. Petrographical features of the Séguéla kimberlites are those of South Africa Group-II kimberlites.

The major element contents are strongly modified by talcification process and alteration (Table 1). But trace elements confirm the Group II assigning, as shown by the Nb/La-vs.-Ce/Sr diagram of Taylor et al. [15]. Incompatible elements are enriched and fractionated $\left((\mathrm{La} / \mathrm{Yb})_{\mathrm{N}}=86-232\right)$ except $\mathrm{Rb}, \mathrm{Ba}$ and $\mathrm{Sr}$, which are mobile in water (Fig. 3). However, $\mathrm{Nb}$ and Th are not enriched compared to $\mathrm{La}\left((\mathrm{Nb} / \mathrm{La})_{\mathrm{N}}=1.05-0.74\right.$; $\left.(\mathrm{Th} / \mathrm{La})_{\mathrm{N}}=1.03-0.50\right)$. Lower trace element contents in the nodule kimberlite are explained by the dilution effect due to the cumulate olivine. The Séguéla kimberlite composition is richer in trace elements than the Kimberley one and close to that of Sierra Leone (Koidu, Fig. 4) but with a lower $\mathrm{Nb} / \mathrm{La}$ ratio $(<1)$ and the lack of $\mathrm{Zr}-\mathrm{Hf}$ negative anomalies. These discrepancies indicate a different source, as shown by the Group I affinity of the Koidu kimberlites [15]. This might be due to the different location: inner part of the lithosphere thick Archaean craton for Koidu, and outer edge of this craton for Séguéla.

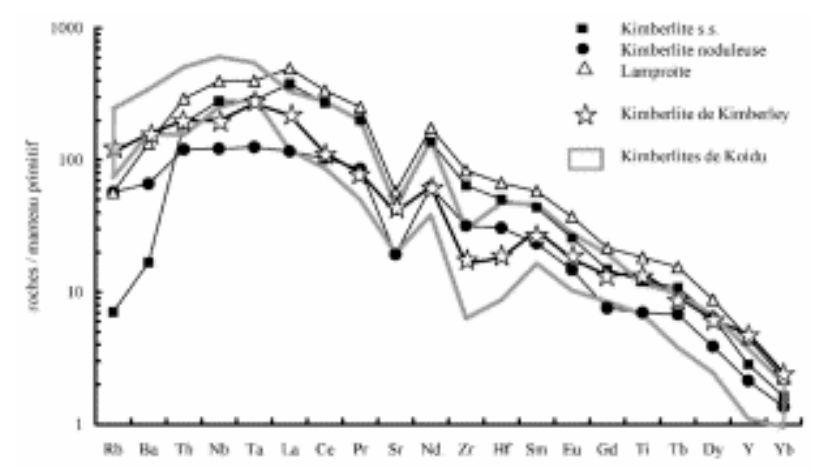

Fig. 3. Diagramme normalisé au manteau primitif des compositions des roches kimberlitiques. Normalisation de Sun et McDonough [14]. Kimberlite de Kimberley [12] ; aire limitée en grisé, kimberlites de Koidu [15].Primitive mantle normalized spiderdiagrams of kimberlitic rocks. Normalization values after Sun and McDonough [14]. Kimberlite of Kimberley [12]; grey dash limited area, kimberlites of Koidu [15]. 


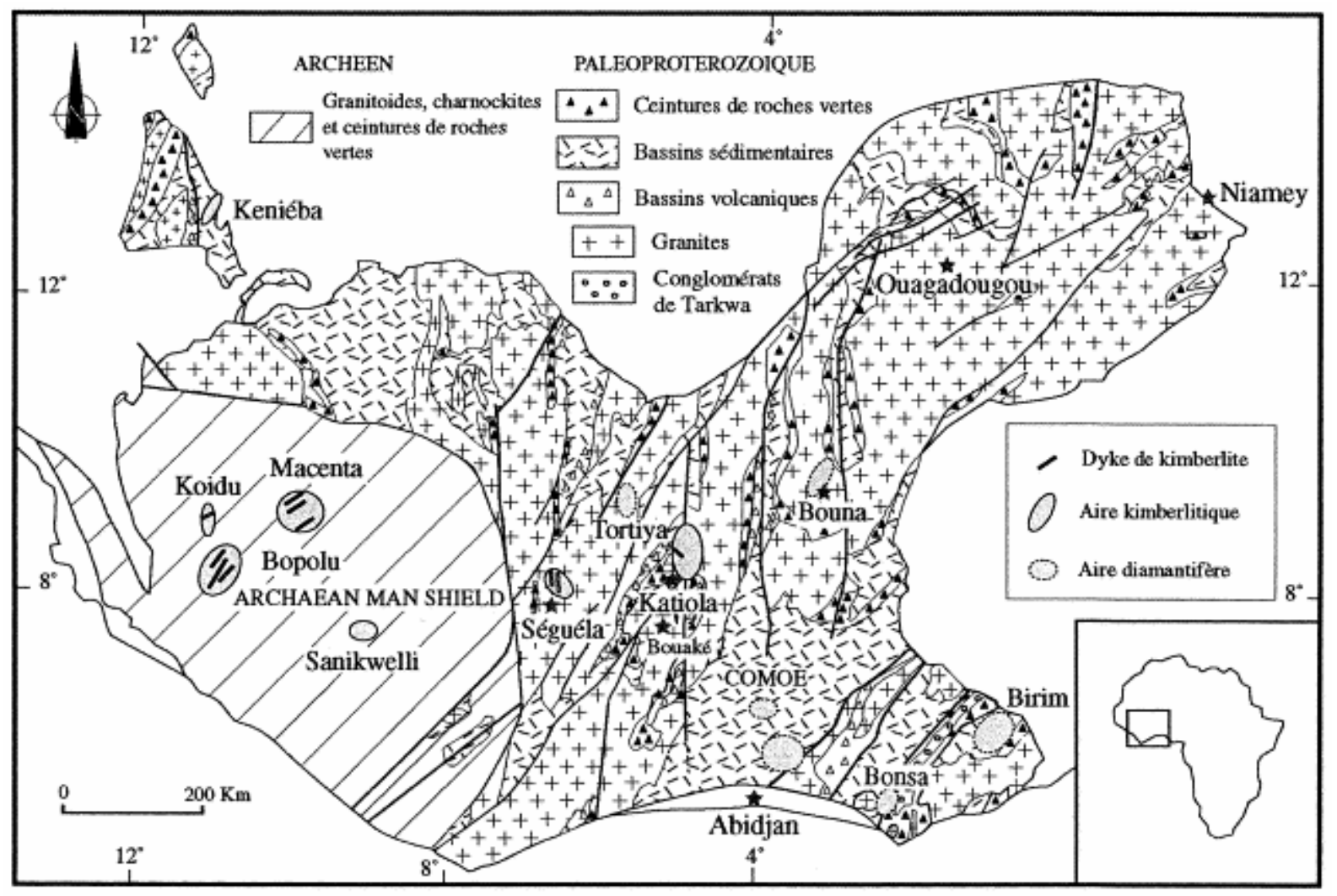

Fig. 4. Localisation des formations kimberlitiques et des aires diamantifères en Afrique de l'Ouest.Location of kimberlitic formations and of diamond-rich area in West Africa.

\section{Presumed age}

Dykes are devoid of any tectonic or metamorphic effect. They were emplaced at shallow depth. The diatreme features and the absence of diagenetic processes in the maar deposit attest for the young geological age of the kimberlitic activity. Previous estimations based on $\mathrm{Sr}$ isotope data would indicate unrealistic ages between 1429 and $1145 \mathrm{Ma}$ [2]. A contamination effect is suspected. In West Africa, there are numerous Precambrian diamond-rich fields, but also some kimberlitic dykes and pipes of Mesozoic age: Cretaceous (Hauterivian-Cenomanian) in Sierra Leone (Koidu), according to $\mathrm{Rb}-\mathrm{Sr}$ and palaeomagnetic data [ $\underline{2}$ and 16 ] ( Fig. 4). The Cretaceous age seems to be the more accurate and could be that of the Séguéla kimberlite. The kimberlite emplacement could be due to reactivation of Precambrian basement major tectonic zones that have opened deep conduits to the upper mantle, in response to the continental drifting related to the Central Atlantic spreading. 


\section{Localisation et contexte géologique}

Un important champ diamantifère est localisé au centre-ouest de la Côted'Ivoire, à $30 \mathrm{~km}$ au nord de Séguéla et à $420 \mathrm{~km}$ d'Abidjan (Fig. 1) [ 1 and $\underline{9}$ ]. Il correspond à un épandage minéralisé, sur $3200 \mathrm{~km}^{2}$, dans des éluvions, colluvions et alluvions, alimentées par deux principaux filons kimberlitiques fertiles : le dyke de Bobi et celui de Toubabouko. Les zones les plus riches, dans et autour des dykes, ont fait l'objet d'exploitations industrielles dans les années 1950-1970. Le gisement continue d'être exploité artisanalement. Il produit des pierres de petite taille, 0,3 ct en moyenne, les plus petites étant de 0,02 ct et les plus grosses (très rares) de 4 ct (27 ct pour la plus grosse), de teinte généralement blanche translucide, parfois jaune chamois à chocolat et, rarement, vert pâle. La principale forme cristalline est l'hexatétraèdre à faces arrondies (70 \%), les autres correspondant à quelques tétraèdres et à des grains très érodés et fragmentés. Les dykes traversent deux plutons de granite, séparés par de petits panneaux d'amphibolite appartenant aux formations birimiennes paléoprotérozoïques du craton ouest-africain. Il s'agit de granites monzonitiques à biotite et hornblende, datés par $\mathrm{Pb} / \mathrm{Pb}$ à $2091 \pm 8 \mathrm{Ma}$ [10].

L'avancement des travaux de fouilles artisanales le long du dyke de Toubabouko nous a permis de découvrir, le 9 février 2002, dans la partie nord du filon, un véritable petit diatrème, avec tous ses caractères structuraux : cratère d'explosion comblé de blocs kimberlitiques emballés dans du matériel granitique pulvérisé et recouvert par les sédiments finement stratifiés d'un maar.

\section{Structures kimberlitiques}

Les structures kimberlitiques de la région de Séguéla sont recouvertes par quelques mètres de colluvions. Les fouilles pour les diamants ont exhumé des roches kimberlitiques. Puis des filons ont été reconnus par des tranchées en 1960, par une prospection électromagnétique de la Sodemi (Société pour le développement minier de la Côte-d'Ivoire) en 1967 et par des sondages de la Waston en 1965 et de la Sodemi en 1995.

Le dyke de Bobi (BD) recoupe le bord ouest du pluton oriental. Il est orienté $\mathrm{N} 170^{\circ}$ avec quelques déviations locales $\mathrm{N} 5^{\circ}$ à $25^{\circ}$ et se poursuit, avec une interruption de $300 \mathrm{~m}$ dans le tiers sud, sur un peu plus de $2,5 \mathrm{~km}$. D'une faible puissance de 25 à $50 \mathrm{~cm}$ et vertical ou irrégulièrement penté de $40^{\circ}$ à $70^{\circ}$ vers l'est, il est constitué de lamproïte et de kimberlite à phlogopite. Deux filons décamétriques $\mathrm{N} 145^{\circ}$ et $\mathrm{N} 30^{\circ}$ ont été dégagés $3 \mathrm{~km}$ plus au sud. Le faisceau d'anomalies électromagnétiques de la prospection géophysique dans le secteur du dyke indique l'existence probable d'un essaim de petits filons sur une aire de $6 \times 4 \mathrm{~km}$ allongée $\mathrm{N} 170^{\circ}$. 
Le dyke de Toubabouko (TD) recoupe le pluton occidental, à une dizaine de kilomètres au nord-ouest de celui de Bobi. Il est orienté $\mathrm{N} 170^{\circ}$, avec des ondulations de $\pm 20^{\circ}$, et se développe sur une longueur de $4,5 \mathrm{~km}$, d'après les anomalies électromagnétiques étroitement axées sur le dyke. Dans les zones dégagées et dans les forages, il montre un pendage de $40^{\circ}$ à $70^{\circ}$ vers l'ouest. Épais de $80 \mathrm{~cm}$ à $1 \mathrm{~m}$, il est parfois dédoublé en deux ou trois filonnets anastomosés. Le cœur du filon est fait d'une kimberlite noduleuse et les bordures d'une kimberlite microgrenue peu porphyrique. Le faciès très micacé est moins fréquent. Les épontes montrent une intense fracturation hydraulique de l'encaissant granitique avec bréchification sur 20 à $50 \mathrm{~cm}$ et fénitisation. La fénitisation se traduit par une rubéfaction sur près d'un mètre (transport de fer) et par le développement de gerbes d'arfvedsonite et de lépidomélane dans le granite bréchique (transport de $\mathrm{Na}$ et hydroxydation).

À moins de $500 \mathrm{~m}$ de la terminaison nord reconnue du dyke, un puits fertile dans la kimberlite a conduit les artisans diamineurs à étendre les fouilles en 2000-2001. Au début de l'année 2002, le chantier s'est agrandi jusqu'à une excavation de $80 \mathrm{~m}$ de diamètre sur $30 \mathrm{~m}$ de profondeur (Fig. 2). Les paliers mettent à jour une brèche de cheminée kimberlitique typique avec, dans le fond, des blocs métriques anguleux ou partiellement arrondis et, sur le pourtour, des blocs de quelques décimètres d'une kimberlite microgrenue aphyrique. Ces blocs sont emballés dans des débris granuleux de granite pegmatitique, mélangés à des fragments de kimberlite. Ce ciment granitique forme des couches stratifiées incurvées entre les gros blocs et s'infiltre dans leurs fractures. Il constitue 30 à 40 $\%$ de la brèche du pourtour. Nous n'avons pas trouvé d'enclaves énallogènes. La kimberlite est fortement altérée en talc et céladonite. Les matériaux granitiques sont des clastes mono- à polycristallins de $2 \mathrm{~mm}$ à $2 \mathrm{~cm}$, de quartz, feldspath potassique, feldspath sodique, muscovite et rare biotite. Ils sont assez peu rubéfiés. Un palier oriental situé à mihauteur comportait, à notre arrivée, une couche horizontale d'argile verte très finement stratifiée, reposant directement sur les gros blocs accumulés dans le fond et s'incurvant en cuvette sur les brèches du flanc oriental. Le matériel argileux est constitué de lamines de kaolinite alternant avec des niveaux riches en fines paillettes dorées de phlogopite transformée en smectites et des grains silteux dispersés de quartz, feldspaths potassiques et sodiques et d'hydroxydes de fer (analyse chimique totale, Tableau 1). Cette couche correspond à un dépôt lacustre calme. En extrapolant vers le centre de l'excavation les strates restant visibles sur le bord oriental, cette couche devait s'étendre sur 1,5 à $2,5 \mathrm{~m}$ d'épaisseur, dans le fond d'une dépression qui a été comblée par des colluvions.

En clair, il s'agit du remplissage sédimentaire classique d'un maar, élément constitutif supérieur d'un diatrème produit par l'explosion d'un pipe au contact de la nappe aquifère. Ce pipe se rapporte à une section élargie du dyke de Toubabouko, qui est donc arrivé à très faible profondeur, à une époque relativement récente, compte tenu de la 
parfaite conservation des structures du diatrème. En effet, ce diatrème renfermait, avant l'intervention humaine, les sédiments intacts du maar, qui reposaient sous une couverture de colluvions. À quelques centaines de mètres au nord du diatrème, deux autres fouilles fertiles montrent des brèches kimberlitiques, qui pourraient également appartenir à de petits diatrèmes. C'est donc toute l'extrémité nord du dyke qui a donné lieu à des manifestations explosives.

\section{Composition des produits kimberlitiques}

Nous distinguons trois types pétrographiques : kimberlite sensu stricto, kimberlite à nodule d'olivine et kimberlite micacée à texture de lamproïte. Quelques rares enclaves de pyroxénites cumulatives à olivine, trouvées par forage du TD, s'ajoutent à la liste pétrographique.

La kimberlite commune est microgrenue aphyrique, en bordure de dyke et dans les petits filons, ou microporphyrique à porphyrique, dans différents secteurs des deux dykes, notamment dans le diatrème. Les phénocristaux sont de l'olivine automorphe entièrement pseudomorphosée en serpentine en profondeur (carottes de forage) et en talc près de la surface (stéatitisation par apport en silice des eaux de drainage). La matrice est constituée de fines paillettes de phlogopite titanée $\left(\mathrm{TiO}_{2}=4,8-5,5 \%\right)$, de grains de magnétite et d'amas d'ilménite manganésifère ( $\mathrm{MnO}=10,9-18,7$ $\%$ ) dans un fond altéré en smectites et talc. Les minéraux magmatiques accessoires sont apatite, titanite, zircon, baddeleyite, monazite, pridérite $(\mathrm{BaO}=2,2-3,8 \%)$, jeppéite $(\mathrm{BaO}=17,3-19,9 \%)$ et... diamant. La minéralogie secondaire comprend $\mathrm{Mg}$-chlorites (penninite), saponite, céladonite, talc, anatase, leucoxène, hydroxydes de fer, calcite et quartz en amas sphérolitiques. Pyroxènes et grenats sont absents. La leucite douteuse signalée dans une lamproïte [9] n'est pas confirmée. Les amphiboles parfois présentes, Mg-actinote et arfvedsonite, proviennent de la fénitisation, qui s'étend dans les brèches kimberlitiques de bordure des dykes. La kimberlite à nodule d'olivine a la même paragenèse que la kimberlite s.s. additionnée de xénocristaux érodés d'olivine, abondante, et de spinelle, plus rare. Les grains arrondis d'olivine (transformée en serpentine et talc) peuvent constituer jusqu'à $50 \%$ de la roche ; leur taille va de 1 à $3 \mathrm{~mm}$, dans le faciès micro-noduleux, à près de $10 \mathrm{~mm}$, dans le faciès noduleux. Les grains de spinelles montrent un cœur de $\mathrm{Mg}$ chromhercynite et une enveloppe de chromite et de Cr-magnétite. C'est le faciès commun au cœur du TD. La kimberlite micacée se distingue par le développement des paillettes de phlogopite, dont la composition est la même que dans la kimberlite s.S., et par la présence de titanates riches en Ba. Elle est fréquente à Bobi, mais aussi présente à Toubabouko. Elle est qualifiée de lamproïte par sa texture lamprophyrique à enchevêtrement intersertal de phlogopite. Pétrographiquement, les kimberlites de Séguéla appartiennent au groupe II des kimberlites micacées sud-africaines. 
Les analyses chimiques des trois faciès pétrographiques sont reportées dans le Tableau 1. La composition en éléments majeurs est modifiée par la talcification de sub-surface, qui affecte surtout les kimberlites noduleuses et par une altération deutéritique importante. La vraie nature de la roche ultrabasique magnésienne apparaît avec la lamproïte peu talcifiée $\left(\mathrm{SiO}_{2}=37 \% ; \mathrm{MgO}=20 \%\right)$. La seule analyse ancienne disponible est comparable [15]. Les variations en éléments majeurs rendent illusoire toute attribution aux groupes chimiques des kimberlites. En revanche, les éléments en trace, sur le diagramme Nb/La vs. Ce/Sr de Taylor et al. [15] montrent une affiliation au groupe II des kimberlites sud-africaines. Les éléments incompatibles sont enrichis et fractionnés $\left((\mathrm{La} / \mathrm{Yb})_{N}=86-232\right)$, excepté les éléments mobiles en présence d'eau et sensibles à l'altération (Rb, Ba, Sr) (Fig. 3). Toutefois, on remarque l'absence d'enrichissement relatif en $\mathrm{Nb}$ et $\mathrm{Th}\left((\mathrm{Nb} / \mathrm{La})_{\mathrm{N}}=1,05-0,74 ;(\mathrm{Th} / \mathrm{La})_{N}=1,03-0,50\right)$. Les teneurs plus faibles en éléments en trace dans la kimberlite noduleuse sont dues à la dilution par accumulation d'olivine. Les kimberlites de Séguéla sont plus enrichies que la kimberlite de Kimberley (Fig. 3). Comparées à celles de Sierra Leone (Koidu, Fig. 4), elles sont un peu plus enrichies, mais avec un rapport $\mathrm{Nb} /$ La plus faible $(<1)$ et sans anomalies négatives de $\mathrm{Zr}-\mathrm{Hf}$, ce qui indique une source différente. De fait, les kimberlites de Koidu sont plus proches du groupe I des kimberlites sudafricaines [15]. Cette différence peut être due au contexte : zone interne du craton archéen à lithosphère froide et épaisse pour Koidu et bordure externe moins épaisse de ce craton pour Séguéla.

\section{Datation possible de l'activité kimberlitique}

Les formations kimberlitiques sont indemnes de toutes recristallisations et déformations métamorphiques. Elles recoupent franchement les formations paléoprotérozoïques du Birimien. On ne décèle aucune fracturation clairement postérieure à la mise en place. Cette mise en place s'est faite à très faible profondeur, d'après l'existence d'au moins un diatrème bien conservé, avec ses argiles de maar non compactées et dépourvues de diagenèse.

Dans un rapport non publié, Bardet et Vachette [2] présentent des mesures isotopiques $\mathrm{Rb} / \mathrm{Sr}$ faites sur deux phlogopites de deux échantillons qui donneraient des âges de 1145 et $1367 \mathrm{Ma}$ et une « isochrone » à $1429 \mathrm{Ma}$. Il est impossible de donner une signification à ces mesures, puisqu'un âge aussi ancien n'est pas compatible avec l'état du diatrème. L'hypothèse d'une contamination est très probable. En Afrique de l'Ouest, il existe des champs diamantifères précambriens, notamment dans des formations détritiques du Birimien, mais aussi des dykes et des pipes datés du Mésozoïque (Fig. 4). Nous pouvons comparer les kimberlites de Séguéla à celles de Guinée (Macenta), de Sierra Leone (Koidu) et du Liberia (Bopolu, Sanikwelli), où des pipes diamantifères sont 
exploités. Une isochrone $\mathrm{Nd}-\mathrm{Sm}$ roche-minéraux a livré un âge de $175 \mathrm{Ma}$ pour une kimberlite de Macenta [6] ]. Toutefois, l'absence de données analytiques laisse un doute sur le cogénétisme des minéraux utilisés. Les kimberlites de Sierra Leone dateraient du Crétacé : $96 \mathrm{Ma}$ (Rb/Sr sur phlogopite, ou $92 \mathrm{Ma}$ sur « isochrone » [2]) ou 90-120 Ma (paléomagnétisme [16]). En revanche, le pipe kimberlitique de Karfur (Nigéria) est attribué au Jurassique (vers $165 \mathrm{Ma}$ ), sur le seul argument de l'âge des basaltes voisins de Runka et des «Youger granites » [17]. De fait, au Mésozoïque, deux importantes phases d'activité tectonomagmatique affectent la plaque africaine. La première, au début du Jurassique, voit la mise en place d'un important magmatisme basaltique, avec de nombreux dykes doléritiques datés vers 200 Ma et interprétés comme les précurseurs de l'ouverture de l'Atlantique central $[\underline{4}, \underline{5}$ and 13]. La seconde, au Crétacé inférieur, correspond à de nouvelles manifestations basiques et à des intrusions alcalines [3] . Elle accompagne l'ouverture du golfe de Guinée et la formation des fossés tectoniques en Afrique de l'Ouest et du centre [7]]. La relation entre l'activité kimberlitique de l'Afrique de l'Ouest et ces deux événements tectonomagmatiques peut être discutée. Au sud-ouest de la Côte-d'Ivoire, l'événement jurassique se traduit par la mise en place de plusieurs dykes doléritiques orientés $\mathrm{N} 130^{\circ}$. Cette direction est différente de celle des dykes kimberlitiques de Séguéla, mais il faut tenir compte du fait que les fractures suivent généralement les zones de faiblesses lithosphériques préexistantes. Or, la région de Séguéla est largement affectée par une fracturation $\mathrm{N} 170^{\circ}$ correspondant à un contact fortement structuré entre la lithosphère archéenne à l'ouest et la lithosphère paléoprotérozoïque à l'est. Toutefois, aucun dyke doléritique n'a été observé en relation directe avec les kimberlites.

Pour l'ensemble des kimberlites de l'Afrique de l'Ouest, Haggerty [ㅁ] propose un modèle de mise en place, dans l'évolution géodynamique régionale liée à l'ouverture de l'Atlantique central. La première manifestation magmato-tectonique correspond aux essaims de dykes doléritiques tholéiitiques, au début du Jurassique. Ces dykes sont principalement orientés WNW-ESE, en cohérence structurale avec les futures marges passives. Par la suite, l'ouverture océanique et le drifting, qui se développent au Jurassique supérieur, doivent impliquer d'énormes contraintes au sein des plaques lithosphériques. Au Crétacé inférieur, ces contraintes sont responsables de la formation du système des rifts entre l'Afrique de l'Ouest et l'Afrique centrale (rifts de la Bénoué et du Ténéré), dans un contexte extensif ouest-est [ $\underline{\underline{Z}}$ and $\underline{11}]$. On note alors l'apparition d'un magmatisme alcalin. Entre ces rifts et les marges océaniques, les paléosutures héritées des événements éburnéens et panafricains sont fragilisées et des fractures profondes sont susceptibles de s'ouvrir au sein du craton. C'est ce qui permettrait le drainage des liquides kimberlitiques, notamment au Crétacé supérieur. La mise en place des kimberlites de Séguéla en dykes $\mathrm{N} 170^{\circ}$ parallèles à la faille de Sassandra, en bordure du bloc éburnéen, s'inscrit dans ce modèle d'évolution magmato-tectonique. 
Un âge Crétacé de ces kimberlites, identique à celui des kimberlites de Sierra Leone (Hauterivien-Cénomanien) et du Libéria, semble donc le plus vraisemblable.

\section{Conclusion}

Des filons kimberlitiques diamantifères indemnes de toute déformation et non métamorphiques traversent les formations cristallines paléoprotérozoïques selon une direction générale $N 170^{\circ}$. Cette orientation est contrôlée par une structuration majeure entre les lithosphères archéenne et paléoprotérozoïque du craton ouest-africain. Les différents types pétrographiques associés, kimberlites micacées communes, kimberlites à nodules d'olivine et kimberlites à phlogopite à faciès de lamproïtes, montrent le même chimisme et se rapportent au groupe II des kimberlites sud-africaines. La découverte d'au moins un diatrème bien conservé avec les brèches de cheminée et le dépôt lacustre d'un maar resté intact sous les colluvions actuelles attestent l'âge géologique récent de l'activité kimberlitique. Cette activité peut être rapportée aux événements tectono-magmatiques du Crétacé, par identification avec la phase kimberlitique de Sierra Leone et du Libéria. Elle résulterait de l'ouverture de profondes fractures lithosphériques héritées de la structuration du craton et réactivées à l'occasion de l'ouverture de l'Atlantique central et du drifting de l'Afrique.

\section{Remerciements}

Nos vifs remerciements vont aux deux correcteurs, Yves Bellion et Hervé Bertrand, dont les critiques constructives ont permis d'améliorer nettement la présentation et la discussion des données de cette note.

\section{Bibliographie}

1. M.G. Bardet, Géologie du diamant, Les gisements kimberlitiques de l'Ouest africain. Mém. BRGM 83 (1974), pp. 178-212.

2. M.G. Bardet, M. Vachette, Détermination d'âges de kimberlites de l'Ouest africain et essai d'interprétation des datations des diverses venues diamantifères dans le monde, BRGM Rapport inédit DS 66 A 59, 1966, 15 $\mathrm{p}$

3. Y. Bellion and G. Crévola, Cretaceous and Cainozoic magmatism of the Sénégal Basin (West Africa): a review. In: A.B. Kampunzu and R.T. Lubala, Editors, Magmatism in Extensional Structural Settings: The Phanerozoic African Plate, Springer-Verlag (1991), pp. 189-208. 
4. H. Bertrand, The Mesozoic tholeiitic province of northwest Africa: a volcano-tectonic record of the early opening of the central Atlantic. In: A.B. Kampunzu and R.T. Lubala, Editors, Magmatism in Extensional Structural Settings: The Phanerozoic African Plate, Springer-Verlag (1991), pp. 147-188.

5. K. Deckart, G. Féraud and H. Bertrand, Age of Jurassic continental tholeiites of French Guyana, Surinam and Guinea: implications for the initial opening of the Central Atlantic Ocean. Earth Planet. Sci. Lett. 150 (1997), pp. 205-220.

6. J.-C. Goujou, D. Thiéblemont, C. Delor, A. Cocherie, A. Lacomme, J.-M. Lafon, M. Tegyey, H. Théveniaut, H. Sall, S. Souaré, J. Touré, M. Bah, A. Baldé, $\mathrm{H}$. Sané, BRGM, DNRGH, Notice explicative de la Carte géologique de la Guinée à 1:200 000, feuille $n^{\circ} 30$, Macenta, ministère des Mines, de la Géologie et de l'Environnement, Conakry, Guinée, 1999, 23 p

7. R. Guiraud and J.-C. Maurin, Early Cretaceous rifts of Western and central Africa: an overview. Tectonophysics 213 (1992), pp. 153-168.

8. S.E. Haggerty, Kimberlites in Western Liberia: an overview of the geological setting in a plate tectonic framework. J. Geophys. Res. 87 B13 (1982), pp. 10811-10826.

9. D. Knopf, Les kimberlites et les roches apparentées de Côte d'Ivoire, Bull. $n^{\circ}$ 3, Direction des Mines et de la Géologie de Côte-d'Ivoire, 1970, Sodemi, Abidjan, Côte-d'Ivoire, 202 p

10. A.N. Kouamelan, Géochronologie et géochimie des formations archéennes et protérozoïques de la Dorsale de Man en Côte-d'Ivoire. Implications pour la transition Archéen-Protérozoïque, thèse, université de Rennes, Mém. Géosciences Rennes n 73, 1996, 289 p

11. J.-C. Maurin and R. Guiraud, Basement control in the development of the Early Cretaceous West and Central African rift system. Tectonophysics 228 (1993), pp. 81-95.

12. Y. Muramatsu, Geochemical investigations of kimberlites from the Kimberley area, South Africa. Geochem. J. 17 (1983), pp. 71-86.

13. S. Nomade, A. Pouclet and Y. Chen, The French Guyana doleritic dykes: geochemical evidence of three populations and new data for the Jurassic Central Atlantic Magmatic Province. J. Geodyn. 34 (2002), pp. 595-614.

14. S.S. Sun and W.F. McDonough, Chemical and isotopic systematics of oceanic basalts: implications for mantle composition and processes. In: 
A.D. Saunders and M.J. Norry, Editors, Magmatism in Ocean Basins, Geol. Soc. London Spec. Pub. N 42 (1989), pp. 313-345.

15. W.R. Taylor, L.A. Tompkins and S.E. Haggerty, Comparative geochemistry of West African kimberlites: evidence for a micaceous kimberlite endmember of sublithospheric origin. Geochim. Cosmochim. Acta 58 (1994), pp. 4017-4037.

16. L.A. Tompkins, The Koidu kimberlite complex, Sierra Leone, West Africa, unpublished M.Sci. thesis, University of Massachusetts, 1983, USA

17. J.B. Wright, Volcanic rocks in Nigeria. In: C.A. Kogbe, Editor, Geology of Nigeria, Elisabethan Lagos, Nigeria (1976), pp. 93-142. 